\title{
The Nonhomomorphicity of Boolean Functions
}

\author{
Xian-Mo Zhang ${ }^{1}$ and Yuliang Zheng $^{2}$ \\ 1 School of Info Tech \& Comp Sci, the University of Wollongong, Wollongong \\ NSW 2522, Australia. xianmo@cs.uow.edu.au \\ 2 School of Comp \& Info Tech, Monash University, McMahons Road, Frankston, \\ Melbourne, VIC 3199, Australia. yuliang@pscit.monash.edu.au \\ URL: http://www.pscit.monash.edu.au/links/
}

\begin{abstract}
We introduce the notion of nonhomomorphicity as an alternative criterion that forecasts nonlinear characteristics of a Boolean function. Although both nonhomomorphicity and nonlinearity reflect a "difference" between a Boolean function and all the affine functions, they are measured from different perspectives. We are interested in nonhomomorphicity due to several reasons that include (1) unlike other criteria, we have not only established tight lower and upper bounds on the nonhomomorphicity of a function, but also precisely identified the mean of nonhomomorphicity over all the Boolean functions on the same vector space, (2) the nonhomomorphicity of a function can be estimated efficiently, and in fact, we demonstrate a fast statistical method that works both on large and small dimensional vector spaces.
\end{abstract}

Key Words: Boolean Functions, Cryptography, Nonhomomorphicity, Nonlinear Characteristics.

\section{Motivation of this Research}

It is known that a function $f$ on $V_{n}$ is affine if and only if $f$ satisfies such property that for any even $k$ with $k \geq 4$,

$$
f\left(u_{1}\right) \oplus \cdots \oplus f\left(u_{k}\right)=0
$$

whenever $u_{1} \oplus \cdots \oplus u_{k}=0$.

In addition, it can be verified that $f$ is affine if and only if there exists an even $k$ with $k \geq 4$ such that II holds whenever $u_{1} \oplus \cdots \oplus u_{k}=0$. Therefore we regard II as a characteristic that is useful in telling a non-affine function from an affine one.

Now consider a non-affine function $f$ on $V_{n}$. Let $k$ be an even with $k \geq 4$ and $\left(u_{1}, \ldots, u_{k}\right)$ be a $k$-tuples with $u_{1} \oplus \cdots \oplus u_{k}=0$. If

$$
f\left(u_{1}\right) \oplus \cdots \oplus f\left(u_{k}\right)=0
$$

then $f$ satisfies the affine property at the particular vector $\left(u_{1}, \ldots, u_{k}\right)$. On the other hand, if

$$
f\left(u_{1}\right) \oplus \cdots \oplus f\left(u_{k}\right)=1
$$


then $f$ behaves in a way that is against the affine property at $\left(u_{1}, \ldots, u_{k}\right)$.

The above observations motivate us to define the number of $k$-tuples of vectors in $V_{n},\left(u_{1}, \ldots, u_{k}\right)$ with $u_{1} \oplus \cdots \oplus u_{k}=0$ such that the affine property II is satisfied, as the homomorphicity of $f$, and furthermore, the number of $k$-tuples of vectors in $V_{n},\left(u_{1}, \ldots, u_{k}\right)$ with $u_{1} \oplus \cdots \oplus u_{k}=0$ such that the affine property II is not satisfied, as the nonhomomorphicity of $f$.

While nonhomomorphicity and nonlinearity are similar to each other in that they both reflect a "distance" between a Boolean function and all the affine functions, the former differentiates itself from the latter in the way the "distance" is measured. Nonhomomorphicity has several interesting properties suggesting that it can serve as a useful nonlinearity indicator: (1) unlike other criteria, we have not only established the tight lower and upper bounds on nonhomomorphicity, but also precisely identified the mean of nonhomomorphicity over all the Boolean functions with the same size, (2) the nonhomomorphicity of a function can be estimated efficiently. In fact, we show a fast statistical method for estimating the nonhomomorphicity of a function. The computing time of the statistical method is not relevant to the dimension (number of variables) of the function. This guarantees that we can use a computer program to analyze Boolean functions of higher dimensions efficiently.

\section{Introduction to Boolean Functions}

Denote by $V_{n}$ the vector space of $n$ tuples of elements from $G F(2)$. The truth table of a function $f$ from $V_{n}$ to $G F(2)$ (or simply functions on $V_{n}$ ) is a $(0,1)$-sequence defined by $\left(f\left(\alpha_{0}\right), f\left(\alpha_{1}\right), \ldots, f\left(\alpha_{2^{n}-1}\right)\right)$, and the sequence of $f$ is a $(1,-1)$-sequence defined by $\left((-1)^{f\left(\alpha_{0}\right)},(-1)^{f\left(\alpha_{1}\right)}, \ldots,(-1)^{f\left(\alpha_{2} n-1\right)}\right)$, where $\alpha_{0}=(0, \ldots, 0,0), \alpha_{1}=(0, \ldots, 0,1), \ldots, \alpha_{2^{n-1}-1}=(1, \ldots, 1,1) . f$ is said to be balanced if its truth table contains an equal number of ones and zeros.

Given two sequences $\tilde{a}=\left(a_{1}, \cdots, a_{m}\right)$ and $\tilde{b}=\left(b_{1}, \cdots, b_{m}\right)$, their componentwise product is defined by $\tilde{a} * \tilde{b}=\left(a_{1} b_{1}, \cdots, a_{m} b_{m}\right)$. In particular, if $m=2^{n}$ and $\tilde{a}, \tilde{b}$ are the sequences of functions on $V_{n}$ respectively, then $\tilde{a} * \tilde{b}$ is the sequence of $f \oplus g$.

Let $\tilde{a}=\left(a_{1}, \cdots, a_{m}\right)$ and $\tilde{b}=\left(b_{1}, \cdots, b_{m}\right)$ be two vectors (or sequences), the scalar product of $\tilde{a}$ and $\tilde{b}$, denoted by $\langle\tilde{a}, \tilde{b}\rangle$, is defined as the sum of the component-wise multiplications. In particular, when $\tilde{a}$ and $\tilde{b}$ are from $V_{m},\langle\tilde{a}, \tilde{b}\rangle=$ $a_{1} b_{1} \oplus \cdots \oplus a_{m} b_{m}$, where the addition and multiplication are over $G F(2)$, and when $\tilde{a}$ and $\tilde{b}$ are $(1,-1)$-sequences, $\langle\tilde{a}, \tilde{b}\rangle=\sum_{i=1}^{m} a_{i} b_{i}$, where the addition and multiplication are over the reals.

A $(1,-1)$-matrix $H$ of order $m$ is called a Hadamard matrix if $H H^{t}=m I_{m}$, where $H^{t}$ is the transpose of $H$ and $I_{m}$ is the identity matrix of order $m$. A Sylvester-Hadamard matrix of order $2^{n}$, denoted by $H_{n}$, is generated by the following recursive relation

$$
H_{0}=1, H_{n}=\left[\begin{array}{rr}
H_{n-1} & H_{n-1} \\
H_{n-1} & -H_{n-1}
\end{array}\right], n=1,2, \ldots
$$


Let $\ell_{i}, 0 \leq i \leq 2^{n}-1$, be the $i$ row of $H_{n}$. Then $\ell_{i}$ is the sequence of a linear function $\varphi_{i}(x)$ defined by the scalar product $\varphi_{i}(x)=\left\langle\alpha_{i}, x\right\rangle$, where $\alpha_{i}$ is the $i$ th vector in $V_{n}$ according to the ascending lexicographic order. (See for instance Lemma 2 of 7 .)

Definition 1. A function $f$ on $V_{n}$ is called an affine function if $f(x)=c \oplus$ $a_{1} x_{1} \oplus \cdots \oplus a_{n} x_{n}$ where and each $a_{j}$ and $c$ are constant in $G F(2)$. In particular, $f$ is called a linear function if $c=0$.

Definition 2. The Hamming weight of a $(0,1)$-sequence $\xi$ is the number of ones in the sequence. Given two functions $f$ and $g$ on $V_{n}$, the Hamming distance $d(f, g)$ between them is defined as the Hamming weight of the truth table of $f(x) \oplus g(x)$, where $x=\left(x_{1}, \ldots, x_{n}\right)$. The nonlinearity of $f$, denoted by $N_{f}$, is the minimal Hamming distance between $f$ and all the affine functions on $V_{n}$, i.e., $N_{f}=\min _{i=1,2, \ldots, 2^{n+1}} d\left(f, \varphi_{i}\right)$ where $\varphi_{1}, \varphi_{2}, \ldots, \varphi_{2^{n+1}}$ are all the affine functions on $V_{n}$.

It is known that the nonlinearity of a function $f$ on $V_{n}$ can be expressed as

$$
N_{f}=2^{n-1}-\frac{1}{2} \max \left\{\left|\left\langle\xi, \ell_{i}\right\rangle\right|, 0 \leq i \leq 2^{n}-1\right\}
$$

where $\xi$ is the sequence of $f$ and $\ell_{0}, \ldots, \ell_{2^{n}-1}$ are the rows of $H_{n}$, namely, the sequences of the linear functions on $V_{n}$. (For a proof of 3 see for instance Lemma 6 of 7 .) In addition, the maximum nonlinearity of a function is $2^{n-1}-$ $2^{\frac{1}{2} n-1}$, namely, $N_{f} \leq 2^{n-1}-2^{\frac{1}{2} n-1}$.

Given a function $f$ on $V_{n}$, a $(1,-1)$ matrix defined by $M=\left((-1)^{f\left(\alpha_{i} \oplus \alpha_{j}\right)}\right)$, where $\alpha_{i}, \alpha_{j} \in V_{n}$ and $0 \leq i, j \leq 2^{n}-1$, is called the $(1,-1)$ incidence matrix, or simply, the matrix of $f$. The following is attributed to R. L. McFarland 2:

$$
M=2^{-n} H_{n} \operatorname{diag}\left(\left\langle\xi, \ell_{0}\right\rangle,\left\langle\xi, \ell_{1}\right\rangle, \ldots,\left\langle\xi, \ell_{2^{n}-1}\right\rangle\right) H_{n}
$$

where $\xi$ be the sequence of function $f$ on $V_{n}, \ell_{i}$ be the $i$ th row of $H_{n}$, and $\operatorname{diag}(a, b, \cdots, c)$ denotes the diagonal matrix whose entries on the diagonal are $a, b, \ldots, c$.

A function $f$ on $V_{n}$ is called a bent function 6 if $\left\langle\xi, \ell_{i}\right\rangle^{2}=2^{n}$ for every $i=0,1, \ldots, 2^{n}-1$, where $\xi$ is the sequence of $f$ and $\ell_{i}$ is a row in $H_{n}$. A bent function on $V_{n}$ exists only when $n$ is a positive even number, and it achieves the highest possible nonlinearity $2^{n-1}-2^{\frac{1}{2} n-1}$.

\section{Homomorphicity and Nonhomomorphicity}

The following lemma is important in this paper, as it explores a characteristic property of affine functions which will be useful in studying nonhomomorphicity.

Lemma 1. Let $f$ be a function on $V_{n}$. Then 
(i) $f$ is an affine function if and only if $f$ satisfies such property that for any even $k$ with $k \geq 4, f\left(u_{1}\right) \oplus \cdots \oplus f\left(u_{k}\right)=0$ whenever $u_{1} \oplus \cdots \oplus u_{k}=0$,

(ii) $f$ is an affine function if and only if there exists an even $k$ with $k \geq 4$ such that $f\left(u_{1}\right) \oplus \cdots \oplus f\left(u_{k}\right)=0$ whenever $u_{1} \oplus \cdots \oplus u_{k}=0$.

Proof. Let $f$ be a function on $V_{n}$. We first prove Part (ii) of the lemma.

Assume that $f$ is affine. By using Definition II it is easy to verify that for any even $k$ with $k \geq 4, f\left(u_{1}\right) \oplus \cdots \oplus f\left(u_{k}\right)=0$ whenever $u_{1} \oplus \cdots \oplus u_{k}=0$. Conversely, assume that there exists an even $k$ with $k \geq 4$ such that $f\left(u_{1}\right) \oplus \cdots \oplus f\left(u_{k}\right)=0$ whenever $u_{1} \oplus \cdots \oplus u_{k}=0$. We now prove that $f$ is affine.

Let $u_{1}$ and $u_{2}$ be any two vectors in $V_{n}$. Obviously, the $k$ vectors $u_{1}, u_{2}$, $u_{1} \oplus u_{2}, 0, \ldots, 0$ satisfy $u_{1} \oplus u_{2} \oplus\left(u_{1} \oplus u_{2}\right) \oplus 0 \oplus \cdots \oplus 0=0$. From the assumption,

$$
f\left(u_{1}\right) \oplus f\left(u_{2}\right) \oplus f\left(u_{1} \oplus u_{2}\right) \oplus f(0) \oplus \cdots \oplus f(0)=0
$$

Consider two cases: $f(0)=0$ and $f(0)=1$.

Case 1: $f(0)=0$. In this case $f(c \alpha)=c f(\alpha)$ holds for any vector $\alpha \in V_{n}$ and any value $c \in G F(2)$. Hence 5 can be rewritten as

$$
f\left(u_{1} \oplus u_{2}\right)=f\left(u_{1}\right) \oplus f\left(u_{2}\right)
$$

where $u_{1}$ and $u_{2}$ are arbitrary.

Let $e_{j}$ denote the vector in $V_{n}$, whose the $j$ th component is one and others are zero. For any fixed value $x_{j}$ in $G F(2), j=1, \ldots, n$, from 6 , $f\left(x_{1} e_{1} \oplus\right.$ $\left.\cdots \oplus x_{n} e_{n}\right)=f\left(x_{1} e_{1}\right) \oplus f\left(x_{2} e_{2} \oplus \cdots \oplus x_{n} e_{n}\right)$ Applying 6 repeatedly, we have $f\left(x_{1} e_{1} \oplus \cdots \oplus x_{n} e_{n}\right)=f\left(x_{1} e_{1}\right) \oplus f\left(x_{2} e_{2}\right) \oplus \cdots \oplus f\left(x_{n} e_{n}\right)$ Note that $f(0)=0$ implies $f(c \alpha)=c f(\alpha)$ where $c$ is any value in $G F(2)$ and $\alpha$ is any vector in $V_{n}$. Hence

$$
f\left(x_{1} e_{1} \oplus \cdots \oplus x_{n} e_{n}\right)=x_{1} f\left(e_{1}\right) \oplus \cdots \oplus x_{n} f\left(e_{n}\right)
$$

From the definition of $e_{j}, x_{1} e_{1} \oplus \cdots \oplus x_{n} e_{n}=\left(x_{1}, \ldots, x_{n}\right)$. On the other hand, if we write $f\left(e_{j}\right)=a_{j}, j=1, \ldots, n$ then $\mathbf{n}$ can be rewritten as $f\left(x_{1}, \ldots, x_{n}\right)=$ $a_{1} x_{1} \oplus \cdots \oplus a_{n} x_{n}$ This proves that $f$ is linear.

Case 2: $f(0)=1$. Set $g(x)=1 \oplus f(x)$. Then $g$ is linear. By using the result in Case $1, g\left(x_{1}, \ldots, x_{n}\right)=b_{1} x_{1} \oplus \cdots \oplus b_{n} x_{n}$ where each $b_{j} \in G F(2)$. Hence $f\left(x_{1}, \ldots, x_{n}\right)=1 \oplus b_{1} x_{1} \oplus \cdots \oplus b_{n} x_{n}$ This proves that $f$ is affine.

We now prove Part (i) of the lemma. Assume that $f$ is affine. From Definition II it is easy to check that for any even $k$ with $k \geq 4, f\left(u_{1}\right) \oplus \cdots \oplus f\left(u_{k}\right)=0$ whenever $u_{1} \oplus \cdots \oplus u_{k}=0$. Conversely, assume $f$ satisfies such property that for any even $k$ with $k \geq 4, f\left(u_{1}\right) \oplus \cdots \oplus f\left(u_{k}\right)=0$ whenever $u_{1} \oplus \cdots \oplus u_{k}=0$. Then from Part (ii) of the lemma, $f$ must be affine.

From the characteristic property shown in Lemma II if a function $f$ on $V_{n}$ satisfies $f\left(u_{1}\right) \oplus \cdots \oplus f\left(u_{k}\right)=0$ for a large number of $k$-tuples $\left(u_{1}, \ldots, u_{k}\right)$ of vectors in $V_{n}$ with $u_{1} \oplus \cdots \oplus u_{k}=0$, then the function behaves more like an affine function. This leads us to introduce a new nonlinearity criterion. 
Notation 1. Let $f$ be a function on $V_{n}$ and $k$ an even with $4 \leq k \leq 2^{n}$. For $c \in G F(2)$, denote by $\mathcal{H}_{f, c}^{(k)}$ the collection of ordered $k$-tuples $\left(u_{1}, \ldots, u_{k}\right)$ of vectors in $V_{n}$ with $u_{1} \oplus \cdots \oplus u_{k}=0$ satisfying $f\left(u_{1}\right) \oplus \cdots \oplus f\left(u_{k}\right)=c$ where $c \in G F(2)$ is constant.

Definition 3. Let $f$ be a function on $V_{n}$ and $k$ an even with $4 \leq k \leq 2^{n}$. For $c \in G F(2)$, we call $\tilde{h}_{f, 0}^{(k)}=\# \mathcal{H}_{f, 0}^{(k)}$, the $k$ th-order homomorphicity of $f$, and furthermore, $\tilde{h}_{f, 1}^{(k)}=\# \mathcal{H}_{f, 1}^{(k)}$, the $k$ th-order nonhomomorphicity of $f$, where \#S denotes the number of elements in a set $S$.

Note that there exist $2^{(k-1) n} k$-tuples of vectors in $V_{n},\left(u_{1}, \ldots, u_{k}\right)$, satisfying $\bigoplus_{j=1}^{k} u_{j}=0$. Hence an interesting fact on $\tilde{h}_{f, c}^{(k)}$ follows:

Lemma 2. Let $f$ be a function on $V_{n}$. Then $\tilde{h}_{f, 0}^{(k)}+\tilde{h}_{f, 1}^{(k)}=2^{(k-1) n}$.

We note that Lemma II cannot be extended to the case of odd $k$. This explains why we have not defined homomorphicity or nonhomomorphicity for an odd order.

\section{Calculations of Nonhomomorphicity}

\subsection{High Order Auto-Correlation}

Recall that the auto-correlation of a function is defined as follows:

Definition 4. Let $f$ be a function on $V_{n}$. For a vector $\alpha \in V_{n}$, denote by $\xi(\alpha)$ the sequence of $f(x \oplus \alpha)$. Thus $\xi(0)$ is the sequence of $f$ itself and $\xi(0) * \xi(\alpha)$ is the sequence of $f(x) \oplus f(x \oplus \alpha)$. Let $\Delta(\alpha)$ be the scalar product of $\xi(0)$ and $\xi(\alpha)$. Namely

$$
\Delta(\alpha)=\langle\xi(0), \xi(\alpha)\rangle
$$

$\Delta(\alpha)$ is called the auto-correlation of $f$ with a shift $\alpha$.

Obviously, $\Delta(\alpha)=0$ if and only if $f(x) \oplus f(x \oplus \alpha)$ is balanced, i.e., $f$ satisfies the propagation criterion with respect to $\alpha$. On the other hand, if $|\Delta(\alpha)|=2^{n}$, then $f(x) \oplus f(x \oplus \alpha)$ is a constant and hence $\alpha$ is a linear structure of $f$.

Next we consider a generalization of the definition for auto-correlation. The generalization will turn out to be a useful tool in studying nonhomomorphic characteristics of functions.

Definition 5. Let $f$ be a function on $V_{n}$ and $\xi=\left(a_{0}, a_{1}, \ldots, a_{2^{n}-1}\right)$ be the sequence of $f$. For a vector $\alpha \in V_{n}$ and an integer $k=2,3, \ldots$, the $k$ th-order auto-correlation of $f$ with a shift $\alpha$, denoted by $\Delta^{(k)}(\alpha)$, is defined as

$$
\Delta^{(2)}(\alpha)=\Delta(\alpha), \Delta^{(k)}(\alpha)=\sum_{j=0}^{2^{n}-1}\left[a_{j} \Delta^{(k-1)}\left(\alpha_{j} \oplus \alpha\right)\right], k=3,4, \ldots
$$

where $\Delta(\alpha)$ is the auto-correlation of $f$ as defined in Definition 4 and $\alpha_{j}$ is the vector corresponding to the integer $j$. 
It is important to point out that nonhomomorphicity, high order auto-correlation and high order derivation introduced in 4 are three completely different concepts. Let $f$ be a function on $V_{n}$. In 4 , the derivation of $f$ at vector $\beta$, denoted by $\Delta_{\beta} f(x)$, is defined as follows

$$
\Delta_{\beta} f(x)=f(x) \oplus f(x \oplus \beta) .
$$

and the $k$ th-order derivation of $f$ at vectors $\beta_{1}, \ldots, \beta_{k}$, denoted by $\Delta_{\beta_{1}, \ldots, \beta_{k}}^{(k)} f(x)$, is defined recursively as

$$
\Delta_{\beta_{1}, \ldots, \beta_{k}}^{(k)} f(x)=\Delta\left(\Delta_{\beta_{1}, \ldots, \beta_{k-1}}^{(k-1)} f(x)\right) .
$$

We can see the $k$ th-order derivation of $f$ at vectors $\beta_{1}, \ldots, \beta_{k}, \Delta_{\beta_{1}, \ldots, \beta_{k}}^{(k)} f(x)$, is itself a function on $V_{n}$. In contrast, both the $k$ th-order nonhomomorphicity and the $k$ th-order auto-correlation of $f$ with a shift $\beta$ are fixed integer values. To examine further how the three concepts differ, consider a bent function $f$ of degree $s$. For $k$ even with $k>s$, the $k$ th-order derivation of $f$ at vectors $\beta_{1}, \ldots, \beta_{k}, \Delta_{\beta_{1}, \ldots, \beta_{k}}^{(k)} f(x)$, is obviously the zero function. In contrast, for the $k$ thorder auto-correlation of $f$, we have $\Delta^{(k)}(0)=2^{-n} \sum_{i=0}^{2^{n}-1}\left\langle\xi, \ell_{i}\right\rangle^{k}=2^{\frac{1}{2} n k}$ (which follows from Corollary II and Lemma 3 to be introduced later on), and for the $k$ th-order nonhomomorphicity of $f$, we have $\tilde{h}_{f, 1}^{(k)}=2^{(k-1) n-1}-2^{\frac{1}{2} n k-1}$, which follows from Theorem 3 in Section 5

To examine the properties of the $k$ th-order auto-correlation $\Delta^{(k)}(\alpha)$, we consider a matrix defined by $\left(\Delta^{(k)}\left(\alpha_{i} \oplus \alpha_{j}\right)\right)$ where $i, j=0,1, \ldots, 2^{n}-1$. Note that the diagonal of the matrix $\left(\Delta^{(k)}\left(\alpha_{i} \oplus \alpha_{j}\right)\right)$ is composed of $2^{n}$ repetitions of $\Delta^{(k)}(0)$. By simple induction on $k$, we have the following result:

Theorem 1. Let $f$ be a function on $V_{n}, M$ be the matrix of $f$ and $\xi$ be the sequence of $f$. Then

$$
\left(\Delta^{(k)}\left(\alpha_{i} \oplus \alpha_{j}\right)\right)=M^{k}=2^{-n} H_{n} \operatorname{diag}\left(\left\langle\xi, \ell_{0}\right\rangle^{k},\left\langle\xi, \ell_{1}\right\rangle^{k}, \ldots,\left\langle\xi, \ell_{2^{n}-1}\right\rangle^{k}\right) H_{n}
$$

where $\ell_{0}, \ell_{1}, \ldots, \ell_{2^{n}-1}$ are the rows of $H_{n}$.

This result shows that the two matrices, $\left(\Delta^{(k)}\left(\alpha_{i} \oplus \alpha_{j}\right)\right)$ and

$$
\operatorname{diag}\left(\left\langle\xi, \ell_{0}\right\rangle^{k},\left\langle\xi, \ell_{1}\right\rangle^{k}, \ldots,\left\langle\xi, \ell_{2^{n}-1}\right\rangle^{k}\right)
$$

are similar in the sense that from the former one can easily find out the latter through the use of $H_{n}$, and vice versa. Furthermore, it is not hard to see that the sum of the entries on the diagonal of $\left(\Delta^{(k)}\left(\alpha_{i} \oplus \alpha_{j}\right)\right)$ is identical to that of $\operatorname{diag}\left(\left\langle\xi, \ell_{0}\right\rangle^{k},\left\langle\xi, \ell_{1}\right\rangle^{k}, \ldots,\left\langle\xi, \ell_{2^{n}-1}\right\rangle^{k}\right)$. In other words,

$$
\sum_{i=0}^{2^{n}-1} \Delta^{(k)}\left(\alpha_{i} \oplus \alpha_{i}\right)=2^{n} \Delta^{(k)}(0)=\sum_{i=0}^{2^{n}-1}\left\langle\xi, \ell_{i}\right\rangle^{k} .
$$

Hence we have proved 
Corollary 1. Let $f$ be a function on $V_{n}, M$ be the matrix of $f$ and $\xi$ be the sequence of $f$. Then $\Delta^{(k)}(0)=2^{-n} \sum_{i=0}^{2^{n}-1}\left\langle\xi, \ell_{i}\right\rangle^{k}$.

For $k=2$, we have $\Delta^{(2)}(0)=2^{n}$. This indicates that Corollary $\mathbf{I}$ embodies Parseval's equation (Page 416 of 5 ) $\sum_{i=0}^{2^{n}-1}\left\langle\xi, \ell_{i}\right\rangle^{2}=2^{2 n}$ as a special case in which $k=2$.

\subsection{Expression of Nonhomomorphicity by Other Indicators}

Recall $\mathbf{3}$ ), the nonlinearity of a function $f$ on $V_{n}$ is related to the maximum $\left|\left\langle\xi, \ell_{i}\right\rangle\right|$, where $\xi$ is the sequence of $f$ and $\ell_{i}$ is the $i$ th row of $H_{n}$. We give a precise expression of nonhomomorphicity by using the same indicator.

Theorem 2. For a function $f$ on $V_{n}$ and $k$ an even with $4 \leq k \leq 2^{n}$. $\tilde{h}_{f, 0}^{(k)}$ and $\tilde{h}_{f, 1}^{(k)}$ can be expressed as follows:

(i) $\tilde{h}_{f, 0}^{(k)}=2^{(k-1) n-1}+\frac{1}{2} \Delta^{(k)}(0)=2^{(k-1) n-1}+2^{-n-1} \sum_{i=0}^{2^{n}-1}\left\langle\xi, \ell_{i}\right\rangle^{k}$

(ii) $\tilde{h}_{f, 1}^{(k)}=2^{(k-1) n-1}-\frac{1}{2} \Delta^{(k)}(0)=2^{(k-1) n-1}-2^{-n-1} \sum_{i=0}^{2^{n}-1}\left\langle\xi, \ell_{i}\right\rangle^{k}$

where $\xi$ is the sequence of $f$ and $\ell_{i}$ denotes the ith row of $H_{n}$.

Proof. We need only to prove that $\tilde{h}_{f, 1}^{(k)}=2^{(k-1) n-1}-\frac{1}{2} \Delta^{(k)}(0)$, as the rest part of the theorem follows from Corollary II and the fact that $\tilde{h}_{f, 0}^{(k)}+\tilde{h}_{f, 1}^{(k)}=2^{(k-1) n}$.

Write $\xi=\left(a_{0}, a_{1}, \ldots, a_{2^{n}-1}\right)$ where each $a_{j}= \pm 1$. Consider $u_{j} \in V_{n}, j=$ $1, \ldots, k$, and $\bigoplus_{j=1}^{k} u_{j}=0$. Clearly, $\bigoplus_{j=1}^{k} f\left(u_{j}\right)=1$ if and only if $\Pi_{j=1}^{k} a_{u_{j}}=-1$ where the subscript $u_{j}$ in $a_{u_{j}}$ is viewed as the integer representation of vector $u_{j}$. It is easy to verify

$$
\frac{1}{2}\left(1-\Pi_{j=1}^{k} a_{u_{j}}\right)=\left\{\begin{array}{l}
1 \text { if } \bigoplus_{j=1}^{k} f\left(u_{j}\right)=1 \\
0 \text { if } \bigoplus_{j=1}^{k} f\left(u_{j}\right)=0
\end{array}\right.
$$

Hence

$$
\begin{aligned}
\tilde{h}_{f, 1}^{(k)} & =\frac{1}{2} \sum_{\bigoplus_{j=1}^{k} u_{j}=0}\left(1-a_{u_{j}} a_{u_{2}} \cdots a_{u_{k}}\right) \\
& =\frac{1}{2} \sum_{u_{1}, \ldots, u_{k-1} \in V_{n}}\left(1-a_{u_{1}} a_{u_{2}} \cdots a_{u_{k-1}} a_{u_{1} \oplus u_{2} \oplus \cdots \oplus u_{k-1}}\right) \\
& =2^{(k-1) n-1}-\frac{1}{2} \sum_{u_{1}, \ldots, u_{k-1} \in V_{n}} a_{u_{1}} a_{u_{2}} \cdots a_{u_{k-1}} a_{u_{1} \oplus u_{2} \oplus \cdots \oplus u_{k-1}} \\
& =2^{(k-1) n-1} \\
& -\frac{1}{2} \sum_{u_{1}, \ldots, u_{k-2} \in V_{n}} a_{u_{1}} a_{u_{2}} \cdots a_{u_{k-2}} \sum_{u_{k-1} \in V_{n}} a_{u_{k-1}} a_{u_{1} \oplus u_{2} \oplus \cdots \oplus u_{u_{k-2}} \oplus u_{k-1}}
\end{aligned}
$$




$$
\begin{aligned}
= & 2^{(k-1) n-1}-\frac{1}{2} \sum_{u_{1}, \ldots, u_{k-2} \in V_{n}} a_{u_{1}} a_{u_{2}} \cdots a_{u_{k-2}} \Delta^{(2)}\left(u_{1} \oplus u_{2} \oplus \cdots \oplus u_{k-2}\right) \\
= & 2^{(k-1) n-1} \\
& -\frac{1}{2} \sum_{u_{1}, \ldots, u_{k-3} \in V_{n}} a_{u_{1}} a_{u_{2}} \cdots a_{u_{k-3}} \sum_{u_{k-2} \in V_{n}} a_{u_{k-2}} \Delta^{(2)}\left(u_{1} \oplus u_{2} \oplus \cdots \oplus u_{k-2}\right) \\
= & 2^{(k-1) n-1}-\frac{1}{2} \sum_{u_{1}, \ldots, u_{k-3} \in V_{n}} a_{u_{1}} a_{u_{2}} \cdots a_{u_{k-3}} \Delta^{(3)}\left(u_{1} \oplus u_{2} \oplus \cdots \oplus u_{k-3}\right) \\
& \vdots \\
= & 2^{(k-1) n-1}-\frac{1}{2} \sum_{u_{1}, u_{2} \in V_{n}} a_{u_{1}} a_{u_{2}} \Delta^{(k-2)}\left(u_{1} \oplus u_{2}\right) \\
= & 2^{(k-1) n-1}-\frac{1}{2} \sum_{u_{1} \in V_{n}} a_{u_{1} \in V_{n}} \sum_{u_{2} \in V_{n}} a_{u_{2}} \Delta^{(k-2)}\left(u_{1} \oplus u_{2}\right) \\
= & 2^{(k-1) n-1}-\frac{1}{2} \sum_{u_{1} \in V_{n}} a_{u_{1} \in V_{n}} \Delta^{(k-1)}\left(u_{1}\right)=2^{(k-1) n-1}-\frac{1}{2} \Delta^{(k)}(0) .
\end{aligned}
$$

This completes the proof.

\section{Bounds on Nonhomomorphicity}

First we introduce Hölder's Inequality 3 that will be used in our discussions on lower and upper bounds. It states that for real numbers $c_{j} \geq 0, d_{j} \geq 0$, $j=1, \ldots, k, p$ and $q$ with $p>1$ and $\frac{1}{p}+\frac{1}{q}=1$, the following is true:

$$
\left(\sum_{j=1}^{k} c_{j}^{p}\right)^{1 / p}\left(\sum_{j=1}^{k} d_{j}^{q}\right)^{1 / q} \geq \sum_{j=1}^{k} c_{j} d_{j}
$$

where the quality holds if and only if there exists a constant $\nu \geq 0$ such that $c_{j}=\nu d_{j}$ for each $j=1, \ldots, k$.

By using Hölder's Inequality, we can prove

Lemma 3. Let $f$ be a function on $V_{n}$ and $k$ an even integer with $k \geq 4$. Then

$$
\sum_{i=0}^{2^{n}-1}\left\langle\xi, \ell_{i}\right\rangle^{k} \geq 2^{n+\frac{1}{2} n k}
$$

where the equality holds if and only if $n$ is even and $f$ is bent.

Armed with the above result, next we show a bound on nonhomomorphicity.

Theorem 3. Let $f$ be a function on $V_{n}$ and $k$ an even integer with $k \geq 4$. Then the following statements hold: 
(i) $\tilde{h}_{f, 1}^{(k)}$ satisfies

$$
2^{(k-1) n-1}-\frac{1}{2}\left(2^{n}-2 N_{f}\right)^{k} \leq \tilde{h}_{f, 1}^{(k)} \leq 2^{(k-1) n-1}-2^{\frac{1}{2} n k-1}
$$

where $N_{f}$ denotes the nonlinearity of $f$,

(ii) An equality in (y) holds if and only if $f$ is bent. In other words, $f$ is bent if and only if

$$
\tilde{h}_{f, 1}^{(k)}=2^{(k-1) n-1}-2^{\frac{1}{2} n k-1} .
$$

Recall that the nonlinearity of a function reaches the minimum nonlinearity if and only if the function is affine while the nonlinearity of a function reaches the maximum nonlinearity if and only if the function is bent. The above theorem shows there exists a consistent relationship between nonlinearity and nonhomomorphicity, especially when the order of nonhomomorphicity is large. Thus, if $\tilde{h}_{f, 1}^{(k)}$ is large, we expect that $f$ is closer to a bent function than to an affine one, and conversely if $\tilde{h}_{f, 1}^{(k)}$ is small, then the function is closer to affine than to bent.

As $\tilde{h}_{f, 0}^{(k)}+\tilde{h}_{f, 1}^{(k)}=2^{(k-1) n}$, we have the following complementary result:

Corollary 2. Let $f$ be a function on $V_{n}$ and $k$ an even integer with $k \geq 4$. Then the following statements hold:

(i) $\tilde{h}_{f, 0}^{(k)}$ satisfies

$$
2^{(k-1) n-1}+2^{\frac{1}{2} n k-1} \leq \tilde{h}_{f, 0}^{(k)} \leq 2^{(k-1) n-1}+\frac{1}{2}\left(2^{n}-2 N_{f}\right)^{k} 2^{(k-1) n-1}
$$

where $N_{f}$ denotes the nonlinearity of $f$,

(ii) An equality in (II) holds if and only if $f$ is bent. In other words, $f$ is bent if and only if

$$
\tilde{h}_{f, 0}^{(k)}=2^{(k-1) n-1}+2^{\frac{1}{2} n k-1} .
$$

A consequence of Theorem 3 and Corollary 2 is

Corollary 3. Let $f$ be a function on $V_{n}$ and $k$ an even integer with $k \geq 4$. Then $\tilde{h}_{f, 0}^{(k)}-\tilde{h}_{f, 1}^{(k)} \geq 2^{\frac{1}{2} n k}$, and the equality holds if and only if $f$ is bent.

An implication of the above corollary is that there exists no function on $V_{n}$ such that $\tilde{h}_{f, 0}^{(k)}=\tilde{h}_{f, 1}^{(k)}$.

\section{Comparing Nonhomomorphicity and Nonlinearity}

A natural question on nonhomomorphicity is how it is related to other nonlinear characteristics, such as nonlinearity which indicates the minimum distance between a particular function and all the affine functions. It turns out that nonhomomorphicity and nonlinearity are two indicators that are not directly comparable. We demonstrate this by inspecting three specific functions $f, g$ and $h$ on $V_{2 s}$ with $s \geq 5$.

Recall that the rows in $H_{s}$, the Sylvester-Hadamard matrix of order $2^{s}$, are denoted by $\ell_{i}, i=0,1, \ldots, 2^{s}-1$. The three functions are defined as follows: 
1. $f$ - the sequence of $f$ is the concatenation of $\ell_{1}, \ell_{2}, \ldots, \ell_{2^{s}-1}$ with $\ell_{1}$ being repeated twice, i.e., $\ell_{1}, \ell_{1}, \ell_{2}, \ldots, \ell_{2}-1$.

2. $g$ - the sequence of $g$ is composed of four repetitions of a bent sequence $\eta$ of length $2^{2 s-2}$, i.e., $\eta, \eta, \eta, \eta$.

3. $h$ - the sequence of $f$ is the concatenation of $\ell_{1}, \ell_{4}, \ldots, \ell_{2^{s}-1}$ with $\ell_{1}$ being repeated four times, i.e., $\ell_{1}, \ell_{1}, \ell_{1}, \ell_{1}, \ell_{4}, \ldots, \ell_{2^{s}-1}$.

By using 3 , we know that the nonlinearities of the three functions are $N_{f}=N_{g}=2^{2 s-1}-2^{s}$, and $N_{h}=2^{2 s-1}-2^{s+1}$.

Consider $k$ even with $k \geq 4$. By Theorem $\boldsymbol{\nu}$ we have the following nonhomomorphic characteristics for the three functions:

$$
\begin{aligned}
& \tilde{h}_{f, 1}^{(k)}=2^{2(k-1) s-1}-2^{-2 s-1}\left(2^{s k+2 s}-2^{s k+s+1}+2^{s k+k+s-1}\right) \\
& \tilde{h}_{g, 1}^{(k)}=2^{2(k-1) s-1}-2^{-2 s-1} \cdot 2^{s k+k+2 s-2} \\
& \tilde{h}_{h, 1}^{(k)}=2^{2(k-1) s-1}-2^{-2 s-1}\left(2^{s k+2 s}-2^{s k+s+2}+2^{s k+2 k+s-2}\right)
\end{aligned}
$$

Thus for these three functions $f, g$ and $h$, their nonlinearities and nonhomomorphic characteristics are related as follows:

(i) $f$ v.s. $g$ : $N_{f}=N_{g}$, but $\tilde{h}_{f, 1}^{(k)}>\tilde{h}_{g, 1}^{(k)}$.

(ii) $f$ v.s. $h: N_{f}>N_{h}$, and $\tilde{h}_{f, 1}^{(k)}>\tilde{h}_{h, 1}^{(k)}$.

(iii) $g$ v.s. $h$ : $N_{g}>N_{h}$, but $\tilde{h}_{g, 1}^{(k)}<\tilde{h}_{h, 1}^{(k)}$ if $k \leq s-1$, and $\tilde{h}_{g, 1}^{(k)}>\tilde{h}_{h, 1}^{(k)}$ if $k \geq s$.

Properties of these three functions clearly show that nonlinearity and nonhomomorphicity are not comparable indicators. They, however, can be used to complement each other in studying cryptographic properties of functions.

The two functions $g$ and $h$ are of particular interest: while $\tilde{h}_{g, 1}^{(k)}<\tilde{h}_{h, 1}^{(k)}$ for $k \leq s-1$, their positions are reversed for $k \geq s$. This motivates us to examine the behavior of nonhomomorphicity as $k$ becomes large.

Theorem 4. Let $f$ and $g$ be two functions on $V_{n}$. If $\tilde{h}_{f, 1}^{k} \neq \tilde{h}_{g, 1}^{k}$, then there is an even positive $k_{0}$, such that $\tilde{h}_{f, 1}^{k}<\tilde{h}_{g, 1}^{k}$ for every even $k$ with $k \geq k_{0}$, or $\tilde{h}_{f, 1}^{k}>\tilde{h}_{g, 1}^{k}$ for every even $k$ with $k \geq k_{0}$.

Assume that $N_{f}>N_{g}$. Then from 31, we have

$$
\max \left\{\left|\left\langle\xi, \ell_{i}\right\rangle\right|, 0 \leq i \leq 2^{n}-1\right\}<\max \left\{\left|\left\langle\eta, \ell_{i}\right\rangle\right|, 0 \leq i \leq 2^{n}-1\right\}
$$

Using a similar proof to that for the above theorem, we can show

Theorem 5. Let $f$ and $g$ be two functions on $V_{n}$. If $N_{f}>N_{g}$, then there is an even positive $k_{0}$, such that $\hbar_{f, 1}^{k}>\hbar_{g, 1}^{k}$ for every even $k$ with $k \geq k_{0}$. 
While Theorem 5 shows that nonhomomorphicity and nonlinearity are consistent when the dimension $k$ is large, the three example functions $f, g$ and $h$, together with Theorems 4 and 5 do indicate that nonhomomorphic characteristics of a function cannot be fully predicted by other cryptographic criteria, such as nonlinearity. Therefore, nonhomomorphicity can serve as another important indicator that forecasts certain cryptographically useful properties of the function.

Comparing (ii) of Theorem 2 and 3 , we find that although both nonlinearity and nonhomomorphicity reflect non-affine characteristics, the former focuses on the maximum $\left|\left\langle\xi, \ell_{i}\right\rangle\right|$ while the latter is more concerned over all $\left|\left\langle\xi, \ell_{i}\right\rangle\right|$.

\section{The Mean of Homomorphicity and Nonhomomorphicity}

Let $f$ be a function on $V_{n}, \chi$ denote an indicator (a criterion or a value), and $\chi_{f}$ denote the indicator of $f$. Note that there precisely $2^{2^{n}}$ functions on $V_{n}$. We are concerned with the mean of the indicator $\chi$ over all the functions on $V_{n}$, denoted by $\bar{\chi}$, i.e. $\bar{\chi}=2^{-2^{n}} \sum_{f} \chi_{f}$.

The upper and lower bounds on $\chi_{f}$ cannot provide sufficient information on the distribution of $\chi$ of a majority of functions. For this reason, we argue that the mean of the indicator $\chi$ over all the functions on $V_{n}$, i.e. $\bar{\chi}=2^{-2^{n}} \sum_{f} \chi_{f}$, should also be investigated. Note that there exist precisely $2^{2^{n}}$ functions with $n$ variables.

Notation 2. Let $O_{k}$ ( $k$ is even) denote the collection of $k$-tuples $\left(u_{1}, \ldots, u_{k}\right)$ of vectors in $V_{n}$ satisfying $u_{j_{1}}=u_{j_{2}}, \ldots, u_{j_{k-1}}=u_{j_{k}}$, where $\left\{j_{1}, j_{2}, \ldots, j_{k}\right\}=$ $\{1,2, \ldots, k\}$. Write $o_{k}=\# O_{k}$.

It is easy to verify

Lemma 4. Let $n$ and $k$ be positive integers and $u_{1} \oplus \cdots \oplus u_{k}=0$, where each $u_{j}$ is a fixed vector in $V_{n}$. Then

$$
f\left(u_{1}\right) \oplus \cdots \oplus f\left(u_{k}\right)=0
$$

holds for every function $f$ on $V_{n}$ if and only if $k$ is even and $\left(u_{1}, \ldots, u_{k}\right) \in O_{k}$.

Lemma 5. In Notation $y$ let $k$ be an even with $2 \leq k \leq 2^{n}$. Then

$$
o_{k}=\sum_{t=1}^{k / 2}\left(\begin{array}{c}
2^{n} \\
t
\end{array}\right) \sum_{p_{1}+\cdots+p_{t}=k / 2, p_{j}>0} \frac{(k) !}{\left(2 p_{1}\right) ! \cdots\left(2 p_{t}\right) !}
$$

Proof. Let $\left(u_{1}, \ldots, u_{k}\right) \in O_{k}$. Then the multiple set $\left\{u_{1}, \ldots, u_{k}\right\}$ can be divided into $t$ disjoint subsets $\Pi_{1}, \ldots, \Pi_{t}$ where $(1) 1 \leq t \leq k,(2)$ each $\Pi_{j}$ is a $2 p_{j}$ $\left(p_{j}>0\right)$ copy of a vector $\beta_{j}$ i.e. $\Pi_{j}=\left\{\beta_{j}, \ldots, \beta_{j}\right\}$ and $\left|\Pi_{j}\right|=2 p_{j}$, (3) $\beta_{j} \neq \beta_{i}$, if $j \neq i,(4)\left\{u_{1}, \ldots, u_{k}\right\}=\Pi_{1} \cup \cdots \cup \Pi_{t}$. 
Note that there exist $\left(\begin{array}{c}2^{n} \\ t\end{array}\right)$ different choices of $t$ distinguished vectors $\beta_{1}, \ldots$, $\beta_{t}$ from $V_{n}$. Arranging each multiple set $\left\{u_{1}, \ldots, u_{k}\right\}$, we obtain precisely $(k)$ !/ $\left(2 p_{1}\right) ! \cdots\left(2 p_{t}\right)$ ! distinguished ordered sets. Note that $2 p_{1}+\cdots+2 p_{t}=k$ and $1 \leq t \leq k / 2$. The proof is completed.

From Lemma 4 if $\left(u_{1}, \ldots, u_{k}\right) \in O_{k}$ then $f\left(u_{1}\right) \oplus \cdots \oplus f\left(u_{k}\right)=0$ holds for every function $f$ on $V_{n}$. Therefore, in this case $f\left(u_{1}\right) \oplus \cdots \oplus f\left(u_{k}\right)=0$ with $u_{1} \oplus \cdots \oplus u_{k}=0$ does not really reflect an affine property. Hence we focus on $\mathcal{H}_{f, 0}^{(k)}-O_{k}$ and $\mathcal{H}_{f, 1}^{(k)}$.

Theorem 6. Let $k$ be an even with $2 \leq k \leq 2^{n}$. Then

(i) the mean of $\tilde{h}_{f, 0}^{(k)}$ over all the functions on $V_{n}$ i.e. $2^{-2^{n}} \sum_{f} \tilde{h}_{f, 0}^{(k)}$, satisfies

$$
2^{-2^{n}} \sum_{f} \tilde{h}_{f, 0}^{(k)}=\frac{1}{2} o_{k}+2^{(k-1) n-1}
$$

where $o_{k}$ is given in Lemma 5

(ii) the mean of $\tilde{h}_{f, 1}^{(k)}$ over all the functions on $V_{n}$ i.e. $2^{-2^{n}} \sum_{f} \tilde{h}_{f, 1}^{(k)}$, satisfies

$$
2^{-2^{n}} \sum_{f} \tilde{h}_{f, 1}^{(k)}=-\frac{1}{2} o_{k}+2^{(k-1) n-1}
$$

Proof. To prove Part (i), we consider two cases for $\left(u_{1}, \ldots, u_{k}\right) \in \mathcal{H}_{f, 0}^{(k)}$.

Case 1: $\left(u_{1}, \ldots, u_{k}\right) \in O_{k}$. From Lemma $4 f\left(u_{1}\right) \oplus \cdots \oplus f\left(u_{k}\right)=0$ holds for every function $f$ on $V_{n}$.

Case 2: $\left(u_{1}, \ldots, u_{k}\right) \in \mathcal{H}_{f, 0}^{(k)}-O_{k}$. Note that $f\left(u_{1}\right) \oplus \cdots \oplus f\left(u_{k}\right)$ takes the value zero and the value one with an equal probability of a half for a random function $f$ on $V_{n}$. Therefore

$$
\begin{aligned}
2^{-2^{n}} \sum_{f} \tilde{h}_{f, 0}^{(k)} & =2^{-2^{n}} \sum_{f} \# O_{k}+2^{-2^{n}} \sum_{f} \#\left(\mathcal{H}_{f, 0}^{(k)}(0)-O_{k}\right)=o_{k}+\frac{1}{2}\left[2^{(k-1) n}-o_{k}\right] \\
& =\frac{1}{2} o_{k}+2^{(k-1) n-1}
\end{aligned}
$$

This proves (i) of the theorem.

Part (ii) can be proven in a similar way, once again by noting that $f\left(u_{1}\right) \oplus$ $\cdots \oplus f\left(u_{k}\right)$ takes the value zero and the value one with an equal probability of a half, for a a random function $f$ on $V_{n}$.

A function whose nonhomomorphicity is larger than the mean, namely $\tilde{h}_{f, 1}^{(k)}>$ $2^{-2^{n}} \sum_{f} \tilde{h}_{f, 1}^{(k)}$, indicates that the function is more nonlinear. The converse also holds. 


\section{Relative Nonhomomorphicity}

The concept of relative nonhomomorphicity introduced in this section is useful for a statistical tool to be introduced later.

Notation 3. Let $k$ be an even with $k \geq 4$ and $R_{k}$ denote the collection of ordered $k$-tuples $\left(u_{1}, \ldots, u_{k}\right)$ of vectors in $V_{n}$ satisfying $u_{1} \oplus \cdots \oplus u_{k}=0$.

We have noticed

$$
\# R_{k}=2^{(k-1) n} \text { and } \#\left(R_{k}-O_{k}\right)=2^{(k-1) n}-o_{k} .
$$

From the proof of Theorem 6 if $\left(u_{1}, \ldots, u_{k}\right) \in R_{s}-O_{k}$ then $f\left(u_{1}\right) \oplus \cdots \oplus f\left(u_{k}\right)$ takes the value zero and the value one with equal probability.

Definition 6. Let $f$ be a function on $V_{n}$ and $k$ be an even with $k \geq 4$. Define the $k$ th-order relative nonhomomorphicity of $f$, denoted by $\rho_{f, 1}^{(k)}$, as $\rho_{f, 1}^{(k)}=\frac{\tilde{h}_{f, 1}^{(k)}}{\#\left(R_{k}-O_{k}\right)}$, i.e. $\rho_{f, 1}^{(k)}=\frac{\tilde{h}_{f, 1}^{(k)}}{2^{(k-1) n-o_{k}}}$.

From Theorem 0 we obtain

Corollary 4. Let $k$ be an even with $2 \leq k \leq 2^{n}$. Then the mean of $\rho_{f, 1}^{(k)}$ over all the functions on $V_{n}$ i.e. $2^{-2^{n}} \sum_{f} \rho_{f, 1}^{(k)}$, satisfies $2^{-2^{n}} \sum_{f} \rho_{f, 1}^{(k)}=\frac{1}{2}$.

From Corollary 4

$\rho_{f, 1}^{(k)}\left\{\begin{array}{l}\geq \frac{1}{2} \text { then the nonhomomorphicity of } f \text { is not smaller than the mean } \\ <\frac{1}{2} \text { then the nonhomomorphicity of } f \text { is smaller than the mean }\end{array}\right.$

In practice, if $\rho_{f, 1}^{(k)}$ is much smaller than $\frac{1}{2}$, then $f$ should be considered cryptographically weak.

\section{Estimating Nonhomomorphicity}

As shown in Theorem 2 the nonhomomorphicity of a function can be determined precisely. In this section, however, we introduce a statistical method to estimate nonhomomorphicity. Such a method is useful in fast analysis of functions.

Denote a real-valued $(0,1)$ function on $R_{k}-O_{k}, t\left(u_{1}, \ldots, u_{k}\right)$, as follows

$$
t\left(u_{1}, \ldots, u_{k}\right)=\left\{\begin{array}{l}
1, \text { if } f\left(u_{1}\right) \oplus \cdots \oplus f\left(u_{k}\right)=1 \\
0, \text { otherwise }
\end{array}\right.
$$

Hence from the definition of nonhomomorphicity we have

$$
\tilde{h}_{f, 1}^{(k)}=\sum_{\left(u_{1}, \ldots, u_{k}\right) \in R_{k}-O_{k}} t\left(u_{1}, \ldots, u_{k}\right)
$$


Let $\Omega$ be a random subset of $R_{k}-O_{k}$. Write $\omega=\# \Omega$ and

$$
\bar{t}=\frac{1}{\omega} \sum_{\left(u_{1}, \ldots, u_{k}\right) \in \Omega} t\left(u_{1}, \ldots, u_{k}\right)
$$

Note that this is the "sample mean" II. In particular, $\Omega=R_{n}^{(k)}-O_{k}, \bar{t}$ is identified with the "true mean" or "population mean" I , namely, $\rho_{f, 1}^{(k)}$.

Now consider $\sum_{\left(u_{1}, \ldots, u_{k}\right) \in \Omega}\left(t\left(u_{1}, \ldots, u_{k}\right)-\bar{t}\right)^{2}$. We have

$$
\begin{aligned}
\sum_{\left(u_{1}, \ldots, u_{k}\right) \in \Omega}\left(t\left(u_{1}, \ldots, u_{k}\right)-\bar{t}\right)^{2} & =\sum_{\left(u_{1}, \ldots, u_{k}\right) \in \Omega} t^{2}\left(u_{1}, \ldots, u_{k}\right) \\
& -2 \bar{t} \cdot \sum_{\left(u_{1}, \ldots, u_{k}\right) \in \Omega} t\left(u_{1}, \ldots, u_{k}\right)+\omega \bar{t}^{2}
\end{aligned}
$$

Note that $t^{2}\left(u_{1}, \ldots, u_{k}\right)=t\left(u_{1}, \ldots, u_{k}\right)$. From $[\mathbf{I 3}$

$$
\begin{aligned}
\sum_{\left(u_{1}, \ldots, u_{k}\right) \in \Omega}\left(t\left(u_{1}, \ldots, u_{k}\right)-\bar{t}\right)^{2} & =\omega \bar{t}-2 \omega \bar{t}^{2}+\omega \bar{t}^{2}=\omega \bar{t}-2 \omega \bar{t}^{2}+\omega \bar{t}^{2} \\
& =\omega \bar{t}(1-\bar{t})
\end{aligned}
$$

Hence the quantity of $\sqrt{\frac{1}{\omega-1} \sum_{\left(u_{1}, \ldots, u_{k}\right) \in \Omega}\left(t\left(u_{1}, \ldots, u_{k}\right)-\bar{t}\right)^{2}}$, which is called the "sample standard deviation" 1 and is usually denoted by $\mu$, can be expressed as

$$
\mu=\sqrt{\frac{1}{\omega-1} \sum_{\left(u_{1}, \ldots, u_{k}\right) \in \Omega}\left(t\left(u_{1}, \ldots, u_{k}\right)-\bar{t}\right)^{2}}=\sqrt{\frac{\omega \bar{t}(1-\bar{t})}{\omega-1}}
$$

By using (4.4) in Section 4.B of I , the "true mean" or "population mean", $\rho_{f, 1}^{(k)}$, can be bounded by

$$
\bar{t}-Z_{e / 2} \frac{\mu}{\sqrt{\omega}}<\rho_{f, 1}^{(k)}<\bar{t}+Z_{e / 2} \frac{\mu}{\sqrt{\omega}}
$$

where $Z_{e / 2}$ denotes the value $Z$ of a "standardized normal distribution" which to its right a fraction $e / 2$ of the data, $[0$ holds with a probability of $(1-e) 100 \%$ I .

For example,

when $e=0.2, Z_{e / 2}=1.28$, and I6 holds with a probability of $80 \%$, when $e=0.1, Z_{e / 2}=1.64$, and $\mathbf{I 6}$ holds with a probability of $90 \%$, when $e=0.05, Z_{e / 2}=1.96$, and I6 holds with a probability of $95 \%$, when $e=0.02, Z_{e / 2}=2.33$, and 16 holds with a probability of $98 \%$, when $e=0.01, Z_{e / 2}=2.57$, and 16 holds with a probability of $99 \%$, when $e=0.001, Z_{e / 2}=3.3$, and I 6 holds with a probability of $99.9 \%$. 
From 131, $0 \leq \bar{t}<1$ and it is easy to verify that $\mu$ in 15 satisfies $0 \leq \mu \leq$ $\frac{1}{2} \sqrt{\frac{\omega}{\omega-1}}$, This implies that 16 can be simply replaced by

$$
\bar{t}-\frac{Z_{e / 2}}{2 \sqrt{\omega-1}}<\rho_{f, 1}^{(k)}<\bar{t}+\frac{Z_{e / 2}}{2 \sqrt{\omega-1}},
$$

where 17 holds with $(1-e) 100 \%$ probability. Hence if $\omega$ i.e. $\# \Omega$ is large, then the lower bound and the upper bound on $\rho_{f, 1}^{(k)}$ in 16 are closer to each other. On the other hand, if we choose $\omega=\# \Omega$ large enough then $Z_{e / 2} \frac{\mu}{\sqrt{\omega}}$ is sufficiently small, and hence $\mathbf{I 6}$ and $\mathbf{I 0}$ will provide us with useful information. For instance, viewing Corollary $\mathbb{1}$ and [7], we can choose $\omega=\# \Omega$ such that $\frac{Z_{e / 2}}{2 \sqrt{\omega-1}}<10^{-p}$. Hence $\omega \geq Z_{e / 2} \cdot 10^{2 p}$ is large enough. In this case I[7 is specialized as

$$
\bar{t}-10^{-p}<\rho_{f, 1}^{(k)}<\bar{t}+10^{-p}
$$

where 18 holds with $(1-e) 100 \%$ probability.

In summary, we can analyze the nonhomomorphic characteristics of a function on $V_{n}$ in the following steps:

1. we randomly fix even $k$ with $k \geq 4$, for example, $k=4,6$ or 8 , and randomly fix a large integer $\omega$, for example, $\omega \geq Z_{e / 2} \cdot 10^{2 p}$, and randomly choose a subset of $R_{k}-O_{k}$, say $\Omega$, with $\# \Omega=\omega$,

2. by using I3 , we determine $\bar{t}$, i.e. "the sample mean",

3. by using $\mathbb{I 8}$, we determine the range of $\rho_{f, 1}^{(k)}$ with a high reliability,

4. viewing $\rho_{f, 1}^{(k)}$ in 18 , from Corollary 4

$$
\rho_{f, 1}^{(k)}\left\{\begin{array}{l}
\geq \frac{1}{2} \text { then } f \text { is not less nonhomomorphic than the mean } \\
>\frac{1}{2} \text { then } F \text { is less nonhomomorphic than the mean }
\end{array}\right.
$$

where I0 holds with $(1-e) \%$ probability,

5 . if $\rho_{f, 1}^{(k)}$ is much smaller than $\frac{1}{2}$ then $f$ should be considered as cryptographically weak.

We have noticed that the statistical analysis has following advantages:

(1) the relative nonhomomorphicity, $\rho_{f, 1}^{(k)}$ can be precisely identified by the use of "population mean" or "true mean",

(2) by using this method we do not need to search through the entire $V_{n}$,

(3) the method is highly reliable.

\section{Extensions to S-boxes}

Obviously, the concept of nonhomomorphicity of a Boolean function can be extended to that of an S-box in a straightforward way. Analysis of the general 
case of an S-box, however, has turned out to be far more complex. Nevertheless, we have obtained a number of interesting results on S-boxes, some of which encompass results presented in this paper. We will report the new results in a forthcoming paper. In the same paper we will also discuss how to utilize nonhomomorphic characteristics of an S-box employed by a block cipher in analyzing cryptographic weaknesses of the cipher.

\section{Conclusions}

Nonhomomorphicity is a new indicator for nonlinear characteristics of a function. It can complement the more widely used indicator of nonlinearity. Two useful properties of nonhomomorphicity are: (1) the mean of nonhomomorphicity over all the Boolean functions over the same vector space can be precisely identified, (2) the nonhomomorphicity of a function can be estimated efficiently, regardless of the dimension of the vector space.

\section{Acknowledgment}

The first author was supported by a Queen Elizabeth II Fellowship (227 23 1002).

\section{References}

1. Stephen A. Book. Statistics. McGraw-Hill Book Company, 1977.

2. J. F. Dillon. A survey of bent functions. The NSA Technical Journal, pages 191-215, 1972. (unclassified).

3. Friedhelm Erwe. Differential And Integral Calculus. Oliver And Boyd Ltd, Edinburgh And London, 1967.

4. X. Lai. Higher order derivatives and differential cryptanalysis. In Proceedings of the Symposium on Communication, Coding and Cryptography, in the Honor of James L. Massey on the Occasion of his 60's Birthday, pages 227-233. Kluwer Academic Publishers, 1994.

5. F. J. MacWilliams and N. J. A. Sloane. The Theory of Error-Correcting Codes. North-Holland, Amsterdam, New York, Oxford, 1978.

6. O. S. Rothaus. On "bent" functions. Journal of Combinatorial Theory, Ser. A, 20:300-305, 1976.

7. J. Seberry, X. M. Zhang, and Y. Zheng. Nonlinearity and propagation characteristics of balanced boolean functions. Information and Computation, 119(1):1-13, 1995. 